\title{
THE DYNAMICS OF NERVE GROWTH FACTOR-INDUCED NEUROFILAMENT AND VIMENTIN FILAMENT EXPRESSION AND ORGANIZATION IN PC12 CELLS ${ }^{1}$
}

\author{
VIRGINIA M. -Y. LEE ${ }^{2}$ AND CONSTANCE PAGE \\ Division of Neuropathology, Department of Pathology and Laboratory Medicine, University of Pennsylvania School of Medicine, \\ Philadelphia, Pennsylvania 19104
}

Received October 20, 1983; Revised January 24, 1984; Accepted January 30, 1984

\begin{abstract}
Monoclonal antibodies specific for neurofilament (NF) subunits or for vimentin filament (VF) protein were used to study nerve growth factor (NGF)-induced intermediate filament expression and organization in a rat pheochromocytoma cell line (PC12 cells). NGF induced increased amounts of NF subunits and VF protein, and they were, in part, differentially localized within PC12 cells. The relative expression of each of the NF subunits and of the VF protein was measured by an enzyme-linked immunosorbent assay which revealed that PC12 cells grown in the absence of NGF (PC12- cells) contained 3 times more VF than 68,000-dalton NF subunits and only barely detectable amounts of 150,000- and 200,000-dalton NF subunits. Exposure of these cells to NGF (PC12+ cells) increased the amount of 68,000-dalton NF subunits 8 -fold, VF protein 3 -fold, and 150,000- and 200,000 -dalton NF subunits 2 -fold. The ratio of VF to 68,000 -dalton NF proteins in PC12- versus $\mathrm{PC} 12+$ cells decreased from 3.0 to 1.5. Both VF and 68,000-dalton NF subunits were arranged in juxtanuclear "ball-like" configurations and both were present in neurites of PC12+ cells. The distribution of 150,000- and 200,000-dalton NF subunits was diffuse with perinuclear stippling and only occasional, weakly fluorescent "balls." After perturbation of the PC12 cell cytoskeleton, 68,000dalton NF subunits and VF redistributed together and, thus, may exist as heteropolymers. Finally, the increased expression of NF and VF proteins was specific for NGF since they could not be induced by other hormones or "growth" factors.

We conclude that PC12 cells constitute a model system for studies of NF and VF expression, assembly, and interactions.
\end{abstract}

Biochemical studies of neurofilament (NF) proteins are limited by the heterogeneous structure of CNS and PNS tissue. Primary cultures of neurons are homogeneous, but they do not provide the quantities of NF proteins necessary for many kinds of biochemical studies. A clonal, nerve growth factor (NGF)-responsive rat pheochromocytoma (PC12) cell line (Greene and Tischler, $1976,1982)$ has recently been shown to contain NF subunits using NF-specific antisera (Virtanen et al., 1981; Lee et al., 1982a). We have recently developed a library of monoclonal antibodies (MAs) specific for each of the three different intermediate filaments (IFs) of the $\mathrm{CNS}$ and PNS, i.e., glial filaments (GFs), vimentin filaments (VFs), and NF subunits (Lee et al., 1982b, 1984).

\footnotetext{
${ }^{1}$ This work was supported in part by National Institutes of Health Grants NS-18616 and NS-16970, and by March of Dimes Birth Defects Foundation Grant 1-826. W. W. Schlaepfer and J. Q. Trojanowski provided helpful critical comments, and M. A. Obrocka provided expert photographic assistance.

${ }^{2}$ To whom correspondence should be addressed.
}

These antibodies overcome the problem of cross-reacting species in antiserum due to the fact that epitopes of IF classes may be both unique and shared (Pruss et al., 1981), and they also permit the detection and quantitation of small amounts of NF subunits and $\mathrm{VF}^{\mathrm{y}}$ protein. In the studies described here, we used immunochemicals from this library to quantitate the induction of NF and VF protein expression in PC12 cells by NGF over time. We also established that the induction of increased amounts of these IF proteins was specific for NGF. Finally, studies of the interactions of NF and VF proteins in PC12 cells suggested that 68-kilodalton (Kd) NF subunits and VF may exist as heteropolymers. We conclude from these studies that PC12 cells are an important model system for probing the biochemistry and molecular interactions of NF and VF proteins.

\section{Materials and Methods}

PC12 cells in culture. The PC12 cells were grown in plastic culture flasks (Falcon), on glass coverslips or on 
Aclar strips coated with a thin film of air-dried, rat-tail collagen using a culture medium comprised of $85 \%$ RPMI $1640,5 \%$ fetal calf serum, and $10 \%$ heat-inactivated horse serum as previously described (Lee et al,, 1977, 1980, 1981, 1982a). PC12 cells are designated PC12when they are grown in the absence of NGF and PC12+ when grown in the presence of NGF. For experiments using NGF-treated cells (PC12+ cells), $50 \mathrm{ng} / \mathrm{ml}$ of 2.5 S NGF, prepared as described by Mobley et al. (1976), were added to the culture medium.

Monoclonal antibodies (MAs) and monospecific antiserum. A library of MAs has been produced which recognizes unique and shared determinants of each of the subunits of NF as well as unique and shared determinants of VF and GF proteins (Lee et al., 1982b, 1984). The anti-68-Kd MA is designated 2.4H11; and MA designated 4.3F9 recognizes an epitope shared by the 150 $\mathrm{Kd}$ and $200-\mathrm{Kd} \mathrm{NF}$ subunits; the anti-VF MA is designated 1.3H9; and an anti-GF MA, used as a negative control antibody, is designated 2.2B10. Purified bovine NF, VF, and GF were used as the immunogens, and all of the hybridomas are the product of rat-mouse fusions. The details regarding the production and the immunochemical and immunohistochemical characterization of these MAs have been published (Lee et al., 1982b, 1984; Hickey et al., 1983; Trojanowski et al., 1983). For double indirect immunofluorescence experiments, a previously described rabbit anti-68-Kd monospecific antiserum was used (Lee et al., 1982a).

Indirect immunofluorescence studies of PC12 cells. Indirect immunofluorescence was conducted on the $\mathrm{PC12}+$ and PC12- cells as previously described (Lee et al., 1982a). Briefly, prior to each experiment, the cells were rinsed in phosphate-buffered saline (PBS), fixed in acetone at $-20^{\circ} \mathrm{C}$ by immersion for $7 \mathrm{~min}$, and rinsed once more in PBS. Alternatively, the cells were fixed by immersion in freshly prepared $4 \%$ paraformaldehyde in PBS for $10 \mathrm{~min}$ at room temperature and were then permeabilized with a solution of $1 \%$ Triton X-100 in PBS for $1 \mathrm{~min}$ at room temperature followed by a 5-min wash in PBS. This fixation protocol yielded the best results. The fixed cells were then incubated in a given MA (applied as undiluted spent supernatant) for $1 \mathrm{hr}$ at room temperature. After this incubation, they were rinsed for $5 \mathrm{~min}$ with PBS and then incubated for $1 \mathrm{hr}$ in a rhodamine-conjugated goat anti-rat IgG antibody (Cappel). This incubation was terminated by washing the cells in PBS. The strips on which the cells were grown were inverted and mounted onto glass slides with an aqueous mounting medium (Lerner Laboratories) and examined with a Zeiss microscope equipped with epifluorescent ultraviolet illumination and filters for rhodamine viewing.

Perturbation of the cytoskeleton of PC12 cells. To gain further insight into the relationship of NF subunits (especially the 68-Kd NF subunit) and VF, the cytoskeleton of the PC12 cells was manipulated: (1) with colchicine; (2) by replating the cells; (3) by growing the cells in suspension culture; and (4) by seeding the cells at high density.

In the colchicine experiments, PC12+ or PC12- cells were treated for $16 \mathrm{hr}$ with $10 \mu \mathrm{g} / \mathrm{ml}$ of this drug prior to performing indirect immunofluorescence studies following the procedures described above. In the replating experiments, NGF-primed ( 3 weeks in NGF) PC12+ cells were mechanically triturated off the culture dish and resuspended in medium containing NGF. The cells were then replated onto collagen-coated coverslips, and indirect immunofluorescence studies were performed as described above. For the experiments on PC12 cells grown in suspension, $\mathrm{PC} 12+$ or PC12- cells were seeded onto naked Petri dishes. Under these circumstances, the cells do not attach to the surface of the Petri dishes. Instead, they grow in unattached clumps which float in the medium. Finally, the possible effects of cell-cell contact on NF and VF interactions were evaluated by seeding the cells at high density on collagen-coated coverslips or strips.

Substitution of other growth factors for NGF in the PC12 culture medium. In order to eliminate the possibility that the observed changes in NF and VF synthesis and organization were nonspecific and unrelated to NGF, indirect immunofluorescence studies were conducted on PC12 cells which had been grown for up to 1 week in each of the following commercially available hormones or growth factors: insulin ( 20 units $/ \mathrm{ml}$ ), epidermal growth factor $(1 \mu \mathrm{g} / \mathrm{ml})$, dibutyryl cyclic AMP $(200 \mu \mathrm{g} /$ $\mathrm{ml})$, and hydrocortisone $(20 \mu \mathrm{g} / \mathrm{ml})$.

Immunochemical quantitation of $N F$ and $V F$ proteins in PC12 cells. To quantitate the response of the $\mathrm{PC} 12$ cells to NGF with respect to the induction of NF and VF proteins, an enzyme-linked immunosorbent assay (ELISA) was developed. To perform this assay, PC12+ or PC12- cells were plated onto polylysine-coated 96 well culture plates (Costar). At specific time intervals, the monolayer cultures were washed twice with PBS, fixed with $4 \%$ paraformaldehyde in PBS for $10 \mathrm{~min}$, washed twice with PBS, and then permeabilized with $1 \%$ Triton X-100 in PBS for $1 \mathrm{~min}$. Immediately thereafter, the cells were washed again and nonspecific sites were blocked for 30 min with $10 \%$ horse serum in PBS. After this blocking step, different MAs at predetermined dilutions were applied in a total volume of $100 \mu \mathrm{l}$ to each of the wells, and a 2 -hr incubation was performed at room temperature. At the conclusion of this incubation, the cells in each of the wells were washed three times with PBS, and a peroxidase-labeled rabbit anti-rat IgG antibody (Cappel) diluted 1:800 was applied in a volume of $100 \mu \mathrm{l}$ to each of the wells for a second 2 -hr incubation. Unbound antibody was washed from the wells with PBS, and bound labeled antibody was visualized using the chromogen ortho-phenyldiamine in citrate buffer with hydrogen peroxide as previously described (Lee et al., 1984).

The amount of reaction product in the incubation medium was then quantitated by transferring the incubation medium to a second, clean 96-well plate and reading the optical density at $450 \mathrm{~nm}$ on a Multiscan Spectrophotometer. The protein concentration in each of the wells was determined separately on sister cultures of PC12 cells which were processed in an identical manner as the experimental plates, except that the blocking steps and antibody incubation steps were omitted to avoid adding exogenous proteins to the wells. The quan- 
titative results derived from the ELISA were then expressed as activity $/ 30 \mu \mathrm{g}$ of total protein of the PC12+ or PC12- cells. Each experiment was performed in quadruplicate samples and each experiment was repeated at least three times. The average of all experiments and samples was reported.

Controls. In the indirect immunofluorescence studies, MAs directed against different IF proteins served as controls for each other since they recognize different classes of IF proteins. In addition, an anti-GF MA (designated 2.2B10) was used in parallel with the anti-NF subunit MAs and the anti-VF MA in both the indirect immunofluorescence studies and the ELISA studies. GF is present in CNS glia and is not found in PC12 cells. Therefore, the anti-GF MA served as a negative control antibody for the studies of NF and VF proteins. Finally, as positive control tissue in the immunofluorescence studies, acetone-fixed frozen sections of rat cerebellum or peripheral nerve were examined in parallel with the PC12 cells.

\section{Results}

Localization of NF and VF proteins in PC12 cells by immunofluorescence. Data regarding the localization of $\mathrm{NF}$ subunits in the PC12+ and PC12- cells by using our anti-NF subunit MAs confirmed the results previously obtained using monospecific antisera to individual NF subunits (Lee et al., 1982a). Briefly, the anti-68-Kd MA stained in a "ball-like" manner in the PC12- cells; however, these "balls" were small, weakly fluorescent, and were evident in only about one-third of these cells (Fig. 1a). In contrast, an intensely fluorescent "ball-like" staining pattern was observed in approximately $95 \%$ of the PC12+ cells (Fig. $2 a$ ). In addition, $68 \mathrm{Kd}$ immunoreactivity was observed in $\mathrm{PC} 12+$ cell neurites. Using an MA specific for both the 150 - and $200-\mathrm{Kd}$ NF subunits, a weak and diffuse immunofluorescent staining pattern was observed in both the PC12- (Fig. 1c) and PC12+ (Fig. 2c) cells. A weak "ball-like" staining pattern occasionally was observed in the PC12+ but not in the PC12cells. In addition, both PC12+ and PC12- cells exhibited perinuclear fluorescent stippling with this MA. Identical results were obtained when MAs specific for only the $150-\mathrm{Kd}$ or $200-\mathrm{Kd} \mathrm{NF}$ subunits were used (data not shown). In parallel studies conducted on normal CNS and PNS tissue using the same anti-NF MAs, as well as in previous immunoperoxidase studies (Lee et al., 1982b; Hickey et al., 1983; Trojanowski et al., 1983), neuronal staining was always diffuse, and "ball-like" configurations were never seen. The weak staining of PC12 cells with the MA specific for the 150- and 200-Kd NF subunits most likely reflects the low quantities of these antigens since this MA intensely stains both CNS and PNS neurons and their processes (see also quantitative data below).

The distribution of VF proteins was determined with the anti-VF MA, and the pattern of VF protein immunoreactivity was similar to that observed with the anti$68-\mathrm{Kd} \mathrm{NF}$ subunit MA (Figs. $1 e$ and $2 e$ ). Immunoreactive $\mathrm{VF}$ was seen in juxtanuclear "ball-like" structures in nearly all $\mathrm{PCl} 2$ + cells and in linear profiles within their neurites. Small, VF-containing "ball-like" structures were also seen in the $\mathrm{PC} 12$ - cells, but they were weakly fluorescent and less common. In addition, "coils" or "cage-like" structures comprised of immunoreactive VF proteins were seen (Fig. 2e). When double, indirect immunofluorescence was conducted on the $\mathrm{PC} 12+$ and PC12- cells, using a previously described monospecific anti-68-Kd NF subunit rabbit antiserum (Lee et al., 1982a) and the anti-VF MA, VF and $68 \mathrm{Kd}$ immunoreactivity were seen in the same "ball-like" structures (data not shown). The "coils" or "cage-like" structures were stained only by the anti-VF MA (data not shown).

Redistribution of NF and VF proteins in PC12 cells after colchicine treatment. Further evidence that VF protein and the 68-Kd NF subunit were localized in the same domain from which the 150- and 200-Kd NF subunits were excluded was derived from experiments in which the PC12 cells were treated with colchicine $(10$ $\mu \mathrm{g} / \mathrm{ml}$ ) for $16 \mathrm{hr}$ before the immunofluorescence studies. Under these experimental conditions, the "ball-like" structures completely disappeared and both $68-\mathrm{Kd} \mathrm{NF}$ and VF protein immunoreactivity were arranged in "cables" or "coils" (Figs. 1, $b$ and $f$, and 2, $b$ and $f$ ). This was the case in both the $\mathrm{PC} 12+$ and $\mathrm{PC} 12-$ cells. Whether these two different proteins are present as copolymers within the same filaments or in separate filamentous structures occurring in the same location could not be determined in these experiments. However, double, indirect immunofluorescence studies, conducted as described above, demonstrated both of these proteins in the same "cables" or "coils" (data not shown), suggesting that VF and $68-\mathrm{Kd} \mathrm{NF}$ subunits are associated or linked together.

In contrast, parallel studies using $150-$ and $200-\mathrm{Kd}$ specific MAs demonstrated a fine fibrillary staining network in $\mathrm{PC} 12+$ and $\mathrm{PC} 12$ - cells which was distinctly different from the pattern just described (Figs. $1 d$ and $2 d)$. The staining intensity observed with these MAs was increased compared with that observed in PC12 cells not treated with colchicine. This could be due to colchicineinduced aggregation of nonfilamentous $150-$ and $200-\mathrm{Kd}$ NF subunits, and it further distinguishes these two NF subunits from the 68-Kd NF subunit and VF protein.

Time course of NF and VF protein induction with NGF in PC12 cells. As noted earlier, in the absence of NGF, the "balls" containing immunoreactive $68-\mathrm{Kd}$ NF sub-" units and VF were small or absent in the majority of PC12 cells (Fig. 3a). After 1 day in NGF, the PC12 cells displayed a slight increase in staining intensity (Fig. $3 b$ ). By 4 days, $\sim 50 \%$ of the PC12 cells contained mediumsize "balls" comprised of $68-\mathrm{Kd}$ NF subunits and VF proteins (Fig. 3c). By 9 days, over $90 \%$ of the cells contained these "ball-like" structures which filled nearly the entire perikaryal compartment outside the nucleus (Fig. 3d). The only change observed after further NGF exposure (up to 3 weeks) was an increase in staining intensity. Parallel studies conducted at the same time intervals using the MAs specific for the $150-\mathrm{Kd}$ or 200 Kd NF subunits revealed a less dramatic but detectable increase in staining intensity with occasional but weakly staining "ball-like" configurations. 

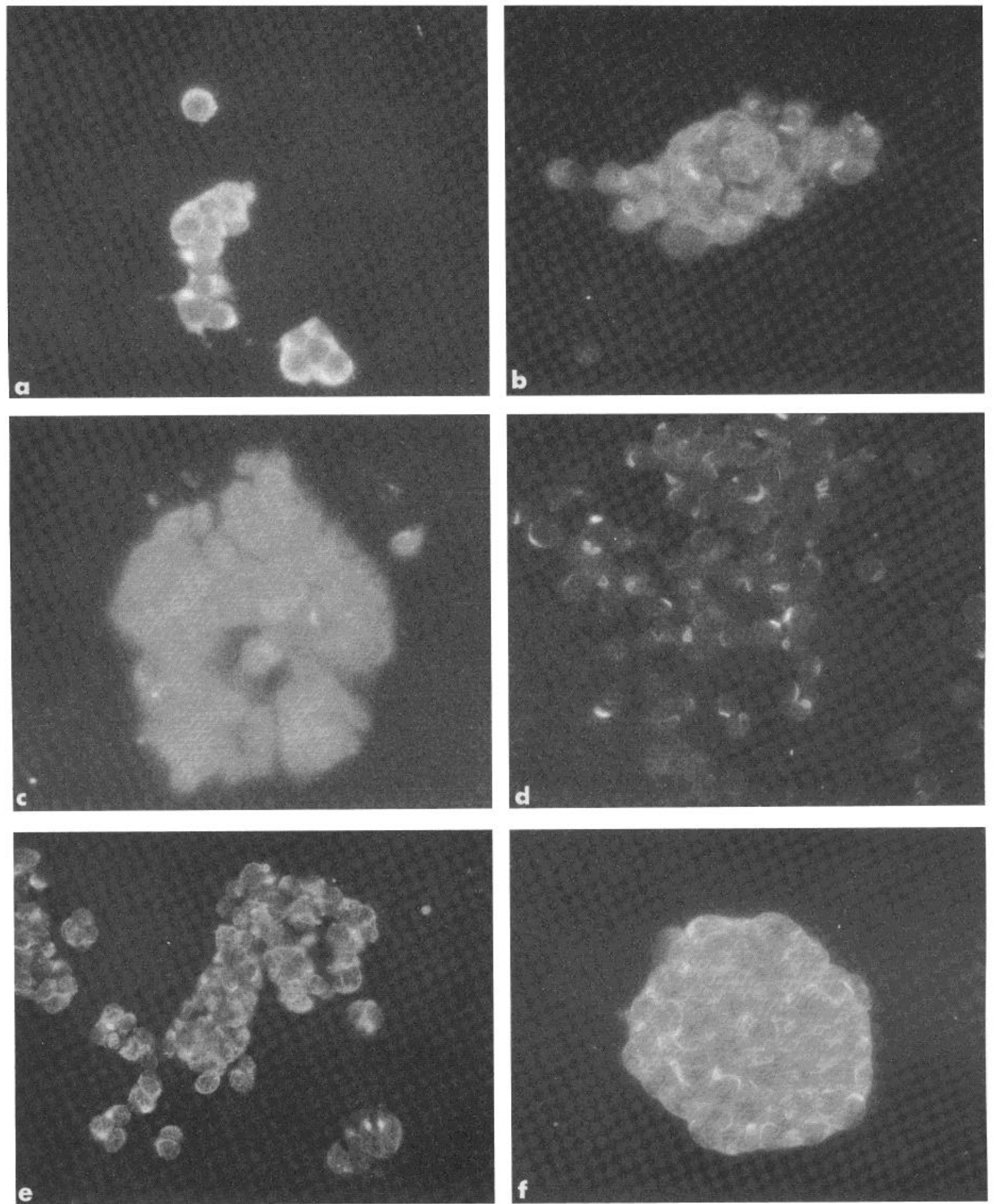

Figure 1. PC12- cells on which immunofluorescence was performed using MAs specific for the 68-Kd NF subunit $(a$ and $b)$, for both the 150- and 200-Kd NF subunits ( $c$ and $d$ ), or for VF protein ( $e$ and $f$ ). The cells illustrated in $b, d$, and $f$ were treated with colchicine prior to the immunofluorescence studies. All photomicrographs were taken with identical exposure times. Magnification $\times 600$.

Effect of replating on $N F$ and VF organization in $P C 12+$ cells. Because cytoskeletal elements are considered to play a role in cell shape and cell motility, we evaluated the effect of replating on NF and VF in NGFprimed ( 3 weeks in NGF) PC12+ cells, using the MAs specific for either the 68-Kd NF subunit or VF. Following the trituration and replating protocol described earlier, indirect immunofluorescence studies were conducted on PC12+ cells at $0,1,2$, and 4 days after replating. On day 0 , "balls" of immunoreactive $68-\mathrm{Kd} \mathrm{NF}$ subunits and VF protein could not be detected (Fig. 4a). One day after replating, small "balls" reappeared (Fig. $4 b$ ) and they 

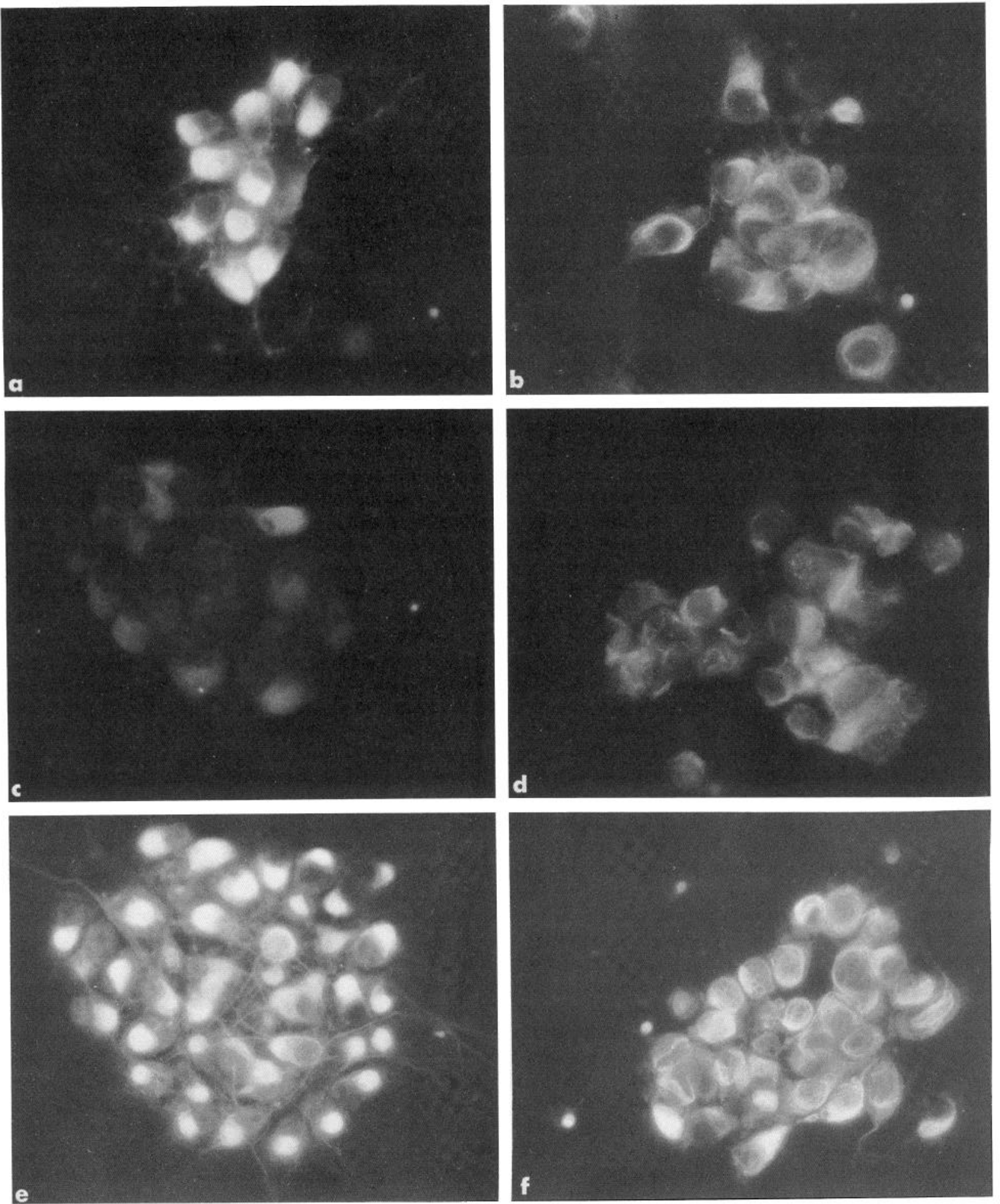

Figure 2. PC12+ cells on which immunofluorescence was performed using MAs specific for the 68-Kd NF subunit $(a$ and $b$ ), for both the 150- and 200-Kd NF subunits $(c$ and $d)$, or for VF protein $(e$ and $f)$. The cells illustrated in $b, d$, and $f$ were treated with colchicine prior to the immunofluorescence studies. All photomicrographs were taken with identical exposure times as in Figure 1. Magnification $\times 600$.

became larger by day 2 (Fig. $4 c$ ). Much larger "balls" of $68-\mathrm{Kd} \mathrm{NF}$ subunits and VF protein were seen at 4 days, and their appearance did not substantially change with longer periods in culture (Fig. $4 d$ ).
Effect of growing PC12 cells at high density or in suspension on NF and VF organization. Both the PC12and PC12+ cells were seeded at high density on Aclar strips. Under these condition, most $\mathrm{PC} 12+$ cells were 

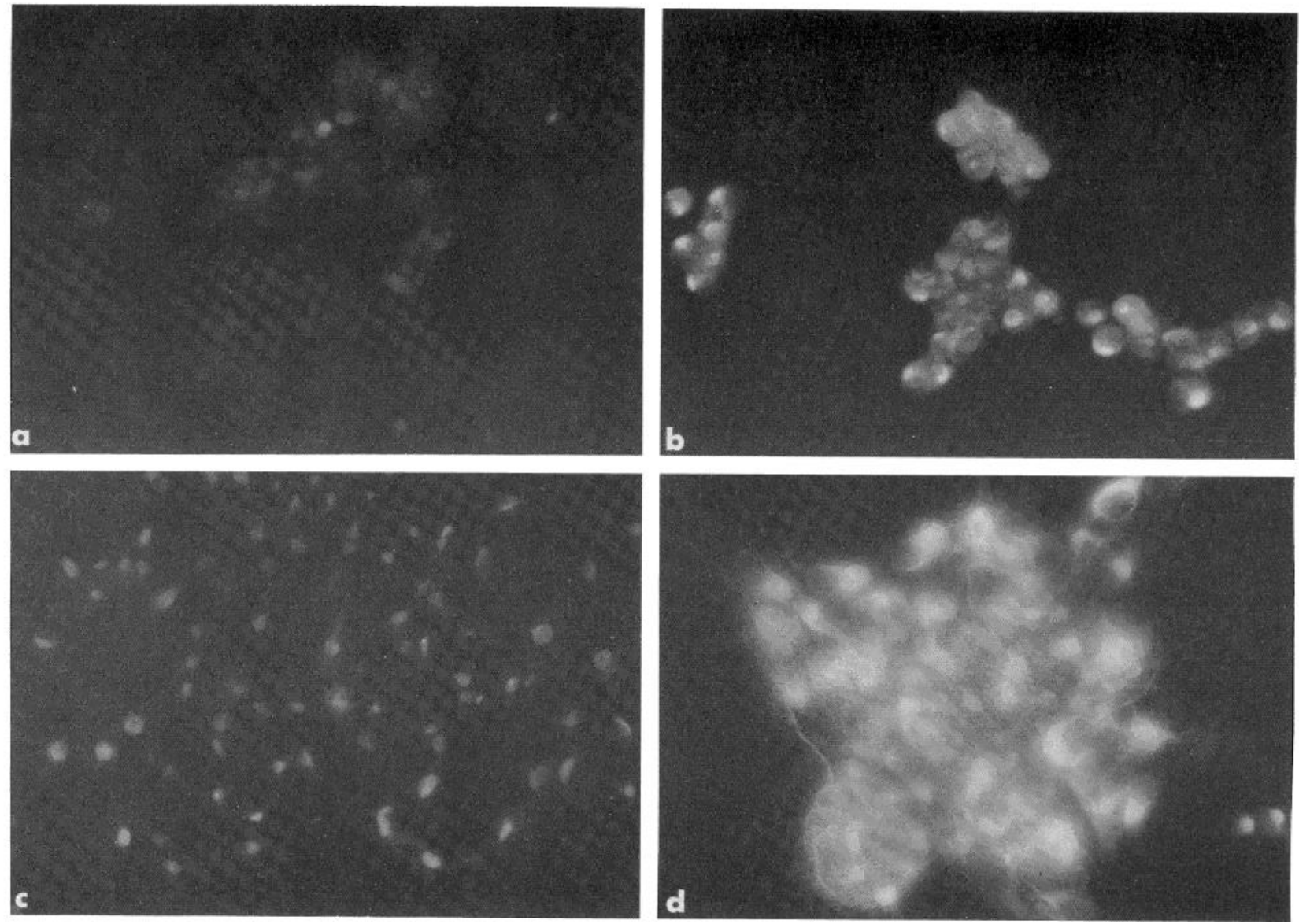

Figure 3. PC12 cells on which immunofluorescence was performed using the MA specific for the 68-Kd NF subunit. The cells in $a$ were grown in the absence of NGF. The cells in $b, c$, and $d$ were exposed to NGF in culture for 1,4 , and 9 days, respectively. All photomicrographs were taken with identical exposure times as in Figures 1 and 2 . Magnification $\times 600$.

devoid of processes. However, in less densely cellular areas, some of the $\mathrm{PC} 12+$ cells had neurites. Indirect immunofluorescence studies of the PC12- cells grown under these conditions produced staining patterns, using each of the MAs described earlier, which were identical to those seen when these cells were not densely seeded (cf. Figs. $1 a$ and Fig. $5 a$ ). In contrast, densely plated PC12+ cells which lacked neurites (comprising the majority of the PC12+ cells grown under these circumstances) contained only small, weakly staining "balls" using the anti-68 Kd-specific MA. At the same time, PC12+ cells with prominent neurites (comprising the minority of PC12+ cells in these experiments) exhibited large and prominent $68 \mathrm{Kd}$-positive "balls" (Fig. $5 b$ ).

Growing the PC12- cells for 1 week in suspension did not significantly alter the pattern of NF or VF protein staining using the respective MAs (Fig. $5 c$ ). However, when PC12+ cells were grown in suspension for the same period of time and examined with the same MAs, marked differences in the organization of NF and VF proteins in these cells were observed by immunofluorescence. The pattern was similar to that just described for $\mathrm{PC} 12+$ cells grown at high density. For example, most of the cells were in clumps and were devoid of neurites. These cells had small, weakly staining "balls" using the MA specific for the 68-Kd NF subunit (Fig. $5 d$ ). About one-third of the $\mathrm{PC} 12+$ cells did display processes and they contained intensely staining, large, 68-Kd NF-positive "balls."

Specificity of the effect of NGF on NF and VF proteins in PC12 cells. To determine whether the effect of NGF on NF and VF proteins in the PC12 cells was specific, we treated PC12 cells for up to 1 week with the following hormones or growth factors: insulin, epidermal growth factor, dibutyryl cyclic AMP, and hydrocortisone. None of these reagents mimicked the effects of NGF on NF and VF proteins in the PC12 cells as detected by indirect immunofluorescence.

ELISA studies on NF and VF proteins in PC12 cells. To provide quantitative data on the increased expression of $\mathrm{NF}$ and VF proteins in PC12 cells by NGF, the relative amounts of these proteins were quantitated by ELISA, using the method described earlier. The results were expressed as activity/30 $\mu \mathrm{g}$ of total protein in the PC12+ or PC12- cells, and these data are summarized in the graphs in Figures 6 and 7. Prior to exposure to NGF, the PC12- cells contained more VF than did all of the NF subunits combined (Fig. 6). The 150- and 200-Kd NF subunits were barely detectable, and there was approximately 3 times more VF than 68 -Kd NF proteins. PC12+ cells grown in NGF for 21 days contained an 8-fold increase in 68-Kd NF subunits compared with PC12cells, whereas the level of VF proteins increased only by 

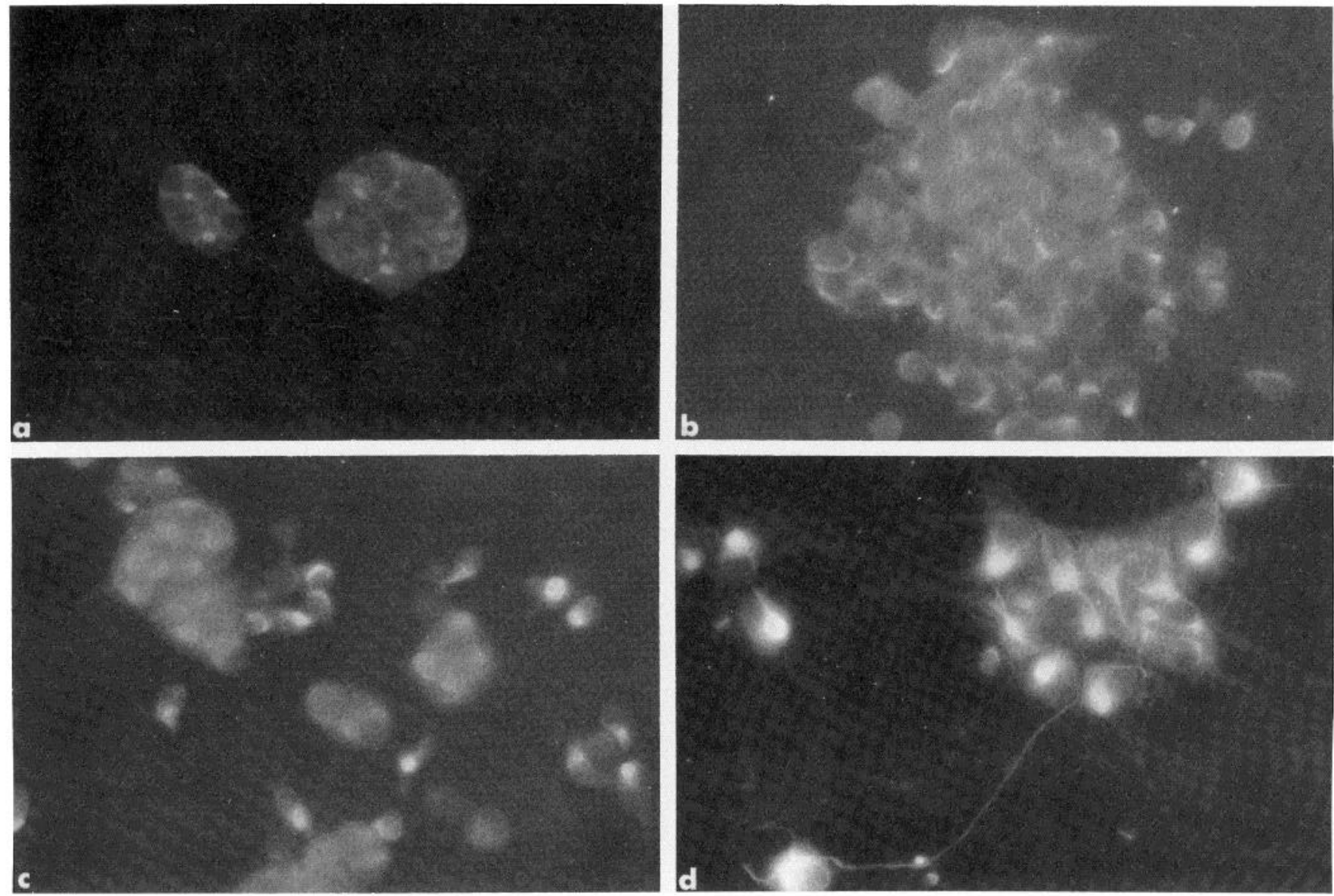

Figure 4. Replated PC12+ (3 weeks in NGF) cells on which immunofluorescence was performed using the MA specific for the 68-Kd NF subunit immediately after mechanical trituration $(a)$ or $1(b), 2(c)$, or $4(d)$ days after trituration and replating. All photomicrographs were taken with identical exposure times as in the previous figures. Magnification $\times 600$.

3 -fold (Fig. 7). The ratio of VF to 68 -Kd NF subunits decreased from $\sim 3.0$ in the PC12- cells to 1.5 in the $\mathrm{PC} 12+$ cells. The amount of 150 - and $200-\mathrm{Kd} \mathrm{NF}$ subunits in $\mathrm{PC} 12+$ cells remained relatively low, but a 2 fold increase was noted.

Using the same quantitative assay, the time course for the induction of NF and VF proteins by NGF in the PC12 cells was also studied. The induction of NF and VF protein synthesis in the $\mathrm{PC} 12+$ cells reached a maximum after 7 days in NGF (Fig. 8). There was only a slight increase in the amount of NF and VF proteins from 7 to 21 days. In addition to using the same MAs in the ELISA studies as those previously employed in the immunofluorescence studies, additional ELISA studies were performed using a rat antiserum rendered monospecific for the 68-Kd NF subunit (Lee et al., 1982a), four other anti-200-Kd NF subunit-specific MAs, and two other anti-150-Kd NF subunit-specific MAs (Lee et al., 1982b). All of these studies yielded results similar to those just described.

\section{Discussion}

Anti-NF subunit-specific MAs were used to establish that $\mathrm{PC} 12$ cells contain each of the three NF subunits. We also demonstrated an NGF-specific induction in the expression of VF and NF proteins by PC12 cells. The ELISA data suggested that the observed differences in immunofluorescence staining intensity reflect quantitative differences in the amounts $\mathrm{NF}$ and VF subunits expressed by $\mathrm{PC} 12+$ and PC12- cells. PC12 cells are therefore an attractive model system for probing the biochemistry and structure of NFs as well as the interactions of NFs with other cytoskeletal components.

The evidence presented here and in two earlier reports (Virtanen et al., 1981; Lee et al., 1982a) is consistent with the hypothesis that PC12 cells are neurons. NF proteins are expressed by cells committed to become neurons (Tapscott et al., 1981). Mammalian NF proteins are found only in neurons (Virtanen et al., 1981; Anderton, 1982; Lazarides, 1982; Schlaepfer, 1983), although an avian NF subunit has recently been described in chicken erythrocytes (Granger and Lazarides, 1983). Reports of other neuron-specific proteins in PC12 cells provide additional evidence that $\mathrm{PC} 12$ cells are committed neurons (Lee et al., 1977, 1980, 1981; Stallcup et al., 1983). To what extent PC12 cells express normal versus aberrant neuronal properties is the subject of continued study (Greene and Tischler, 1982). Other putative neuronal cell lines, such as those derived from neuroblastomas, do not contain NF subunits (Virtanen et al., 1981). Although neuronal differentiation has been induced in embryonal carcinoma cell lines, neuronal cell lines have not yet been established (Jones-Villeneuve et al., 1982).

The genetic and epigenetic mechanisms controlling 

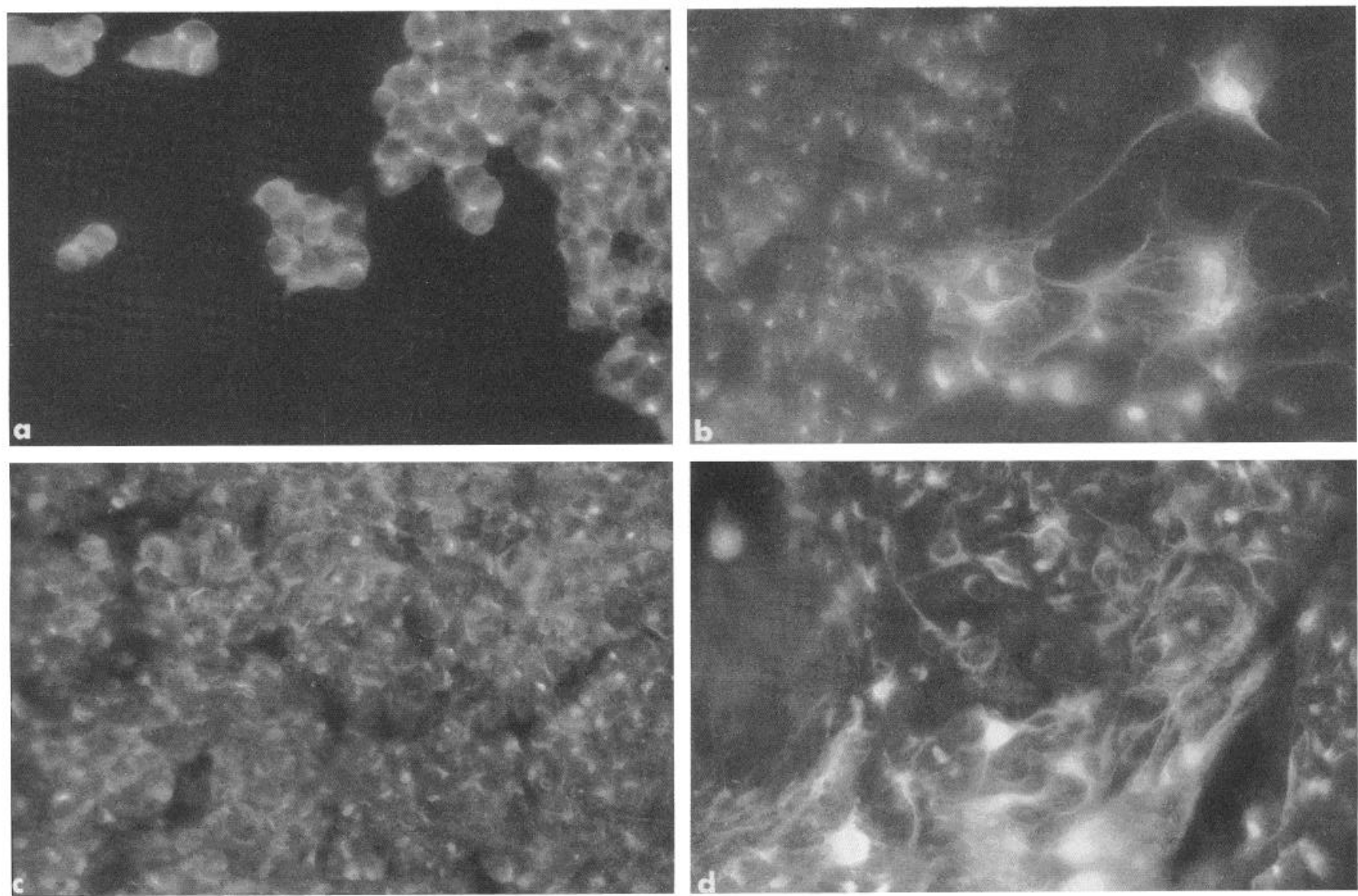

Figure 5. PC12- (a) and PC12+ (b) cells seeded at high cell density and incubated for the immunofluorescent demonstration of the 68-Kd NF subunit. The photomicrographs in $c$ and $d$ are of PC12- and PC12+ cells, respectively, grown in suspension and incubated to demonstrate the $68-\mathrm{Kd} \mathrm{NF}$ subunit. All photomicrographs were taken with identical exposure times as in the previous figures. Magnification $\times 600$.

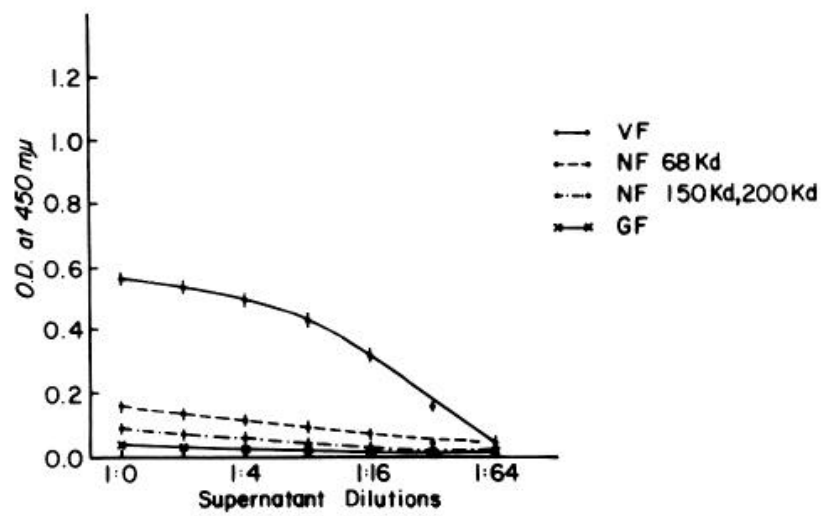

Figure 6. Graph of the relative expression of NF and VF protein in PC12- cells determined by using serial dilutions of specific MA supernatant (horizontal axis). The optical density (O.D.) at each dilution (vertical axis) is expressed per $30 \mu \mathrm{g}$ of total protein of permeabilized PC12- cells per well. Vertical hash marks indicate standard deviations. An anti-GF MA $(2.2 \mathrm{~B} 10)$ was used as a negative control antibody.

NF subunit synthesis, assembly, and turnover remain undefined. The experiments described here regarding the expression of NF subunits by NGF, as well as the modulation of NF protein expression in NGF-primed cells as

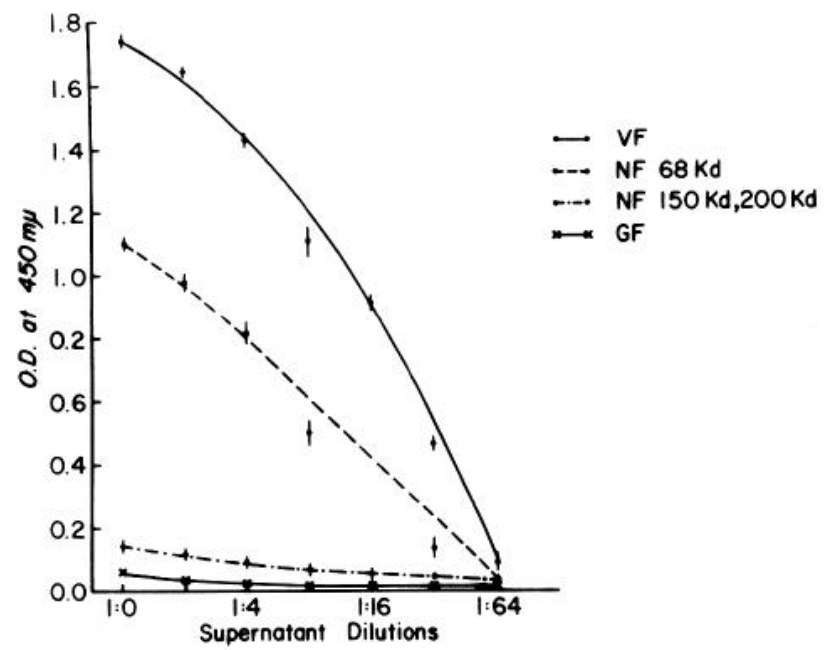

Figure 7. Graph of the relative expression of NF and VF proteins in $\mathrm{PC} 12+$ cells determined and represented as in Figure 6.

a function of replating, are consistent with current hypotheses concerning the mechanisms of action of NGF (Greene and Shooter, 1982). We speculate that the gradual increase in NF subunit expression in PC12 cells 


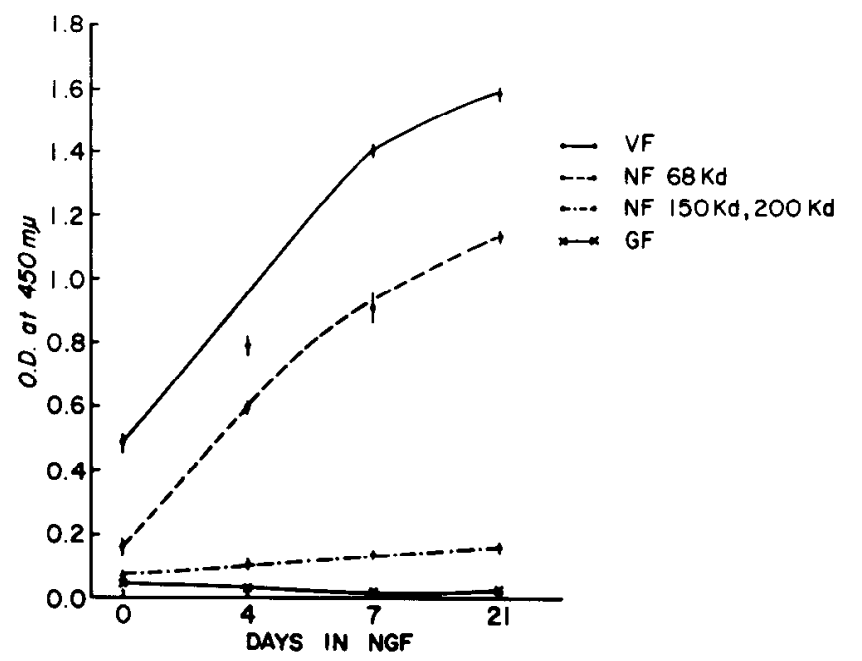

Figure 8. Graph of the time course of NF subunit and VF protein expression in PC12 cells treated with NGF. The time (days in NGF) is expressed on the horizontal axis and the optical density $(O . D$.) per $30 \mu \mathrm{g}$ of total protein of permeabilized $\mathrm{PC} 12$ cells is expressed on the vertical axis. Vertical hash marks indicate the standard deviations.

following exposure to NGF reflects the slow, transcription-dependent effect of this growth factor. In contrast, the rapid increase in NF protein expression in replated PC12+ cells most likely correlates with a transcriptionindependent process. Although NGF is known to be internalized by PC12 cells, its site of action is unknown (Hogue-Angeletti et al., 1983). How NGF affects NF subunit expression is likewise unknown. The putative transcription-independent process regulating NF expression in PC12+ cells may not be an artifact of tissue culture conditions since nerve transection results in increased amounts of immunoreactive NF in anterior horn cells (Moss and Lewkowicz, 1983).

One of the more striking observations in this study was the prominent arrangement of $68-\mathrm{Kd} \mathrm{NF}$ subunits in juxtanuclear "balls." Using double indirect immunofluorescence on $\mathrm{PC} 12+$ cells grown in the presence or absence of colchicine, VFs were seen in the same region as the 68-Kd NF subunits. This result suggests that these two IF proteins are associated or linked. Whether they exist as heteropolymers (Lazarides, 1982; Quinlan and Franke, 1982; Sharp et al., 1982) remains to be determined. Earlier we described juxtanuclear aggregates of 10-nm-diameter filaments in PC12 cells (Lee et al., 1982a), but it was not possible to determine their subunit composition. Immunoelectron microscopic studies will be required in order to resolve these issues. Similar "balls" have not been described in normal CNS or PNS neurons by light or electron microscopy (Anderton, 1982).

We speculate that the aggregation of $\mathrm{VF}$ and $\mathrm{NF}$ subunits into juxtanuclear "balls" and the exclusion of the two higher molecular weight NF subunits from the "ball-like" domain in PC12 cells may be a manifestation of abnormal IF assembly. Mammalian NFs differ from all other IFs in that they have two higher molecular weight subunits associated with the putative "backbone" molecule, i.e., the 68-Kd NF subunit (Wais-Steidler et al., 1983). The exclusion of the 150- and 200-Kd NF subunits from the "ball-like" domain may result from the abnormal association of VF proteins with the 68-Kd NF subunit, thereby preventing this subunit from associating normally with the other two NF triplet proteins. This could lead to "instability" of these structures, resulting in a partial collapse of NF into "balls."

The disappearance and reappearance of these "balls" in PC12 cells as a result of perturbations of the cytoskeleton, growth in suspension and growth in high density cultures, supports the hypothesis that functional interactions occur between IFs and other organelles as well as with the cell surface (Dellagi and Brouet, 1982; Lazarides, 1982; Lehto and Virtanen, 1983; Maro et al., 1983; Menko et al., 1983). These studies also suggest that neurite outgrowth and the appearance of "balls" are related. The increase in the number of "balls" containing 68 -Kd NF subunits, as neurites grow and ramify, may be due to the fact that some of these subunits fail to become properly assembled into intact NFs and instead accumulate in the cytoplasm of PC12 cells. It should be possible to address these and other issues regarding the biology of NFs using PC12 cells as a model system for studies of normal and abnormal aspects of NF expression and organization.

\section{References}

Anderton, B. H. (1982) The neuronal cytoskeleton: Proteins and pathology. In Recent Advances in Neuropathology, W. T. Smith and J. B. Cavanagh, eds., pp. 29-51, Churchill Livingstone, New York.

Dellagi, K., and J. -C. Brouet (1982) Redistribution of intermediate fillaments during capping of lymphocyte surface molecules. Nature 298: 284-286.

Granger, B. L., and E. Lazarides (1983) Expression of the major neurofilament subunit in chicken erythrocytes. Science 221 : $553-556$.

Greene, L. A., and E. M. Shooter (1982) Nerve growth factor: Biochemistry, synthesis and mechanism of action. Annu. Rev. Neurosci. 3: 353-402.

Greene, L. A., and A. S. Tischler (1976) Establishment of a monoadrenergic clonal cell line of rat adrenal pheochromocytoma cells which respond to nerve growth factor. Proc. Natl. Acad. Sci. U. S. A. 73: 2424-2428.

Greene, L. A., and A. S. Tischler (1982) PC12 pheochromocytoma cells in neurobiological research. Adv. Cell. Neurobiol. 3: 373-414.

Hickey, W. F., V. Lee, J. Q. Trojanowski, L. J. McMillan, T. J. McKearn, J. Gonates, and N. K. Gonatas (1983) Immunohistochemical application of monoclonal antibodies against myelin basic protein and neurofilament triplet protein subunits: Advantages over antisera and technical limitations. J. Histochem. Cytochem. 3: 1126-1135.

Hogue-Angeletti, R. A., A. S. Stieber, and N. K. Gonatas (1983) Endocytosis of nerve growth factor by PC12 cells studied by quantitative ultrastructural autoradiography. Brain Res. 241: $145-156$.

Jones-Villeneuve, E. M. V., M. W. McBurney, K. A. Rogers, and V. I. Kalnins (1982) Retinoic acid induces embryonal carcinoma cells to differentiate into neurons and glial cells. J. Cell Biol. 94: 253-262.

Lazarides, E. (1982) Intermediate filaments: A chemically heterogeneous, developmentally regulated class of proteins. Annu. Rev. Biochem. 51: 219-250. 
Lee, V., M. Shelanski, and L. Greene (1977) Specific neural and adrenal medullary antigens detected by antisera to clonal PC12 pheochromocytoma cells. Proc. Natl. Acad. Sci. U. S. A. 74: 5021-5025.

Lee, V., M. Shelanski, and L. Greene (1980) Differential cytotoxic activities of antisera against nerve growth factor treated and untreated clonal pheochromocytoma cells. Neuroscience 5: 1979-1987.

Lee, V., L. Greene, and M. Shelanski (1981) Identification of neural and adrenal medullary surface membrane glycoproteins recognized by antisera to cultured rat sympathetic neurons and PC12 pheochromocytoma cells. Neuroscience 6 : $2773-2786$.

Lee, V., J. Q. Trojanowski, and W. W. Schlaepfer (1982a) Induction of neurofilament triplet proteins in PC12 cells by nerve growth factor. Brain Res. 238: 169-180.

Lee, V. M. -Y., H. -L. Wu, and W. W. Schlaepfer (1982b) Monoclonal antibodies recognize individual neurofilament triplet proteins. Proc. Natl. Acad. Sci. U. S. A. 79: 60896092.

Lee, V. M. -Y., C. D. Page, H. -L. Wu, and W. W. Schlaepfer (1984) Monoclonal antibodies to gel excised glial filament protein and their reactivity with other intermediate filament proteins. J. Neurochem. 42: 25-32.

Lehto, V. -P., and I. Virtanen (1983) Immunolocalization of a novel, cytoskeleton-associated polypeptide of $\mathrm{Mr} 230,000$ (p230). J. Cell Biol. 96: 703-716.

Maro, B., M. -E. Sauron, and M. Bornens (1983) Further evidence for interaction between microtubules and vimentin filaments: Taxol and cold effects. Biol. Cell 47: 243-246.

Menko, A. S., Y. Toyama, D. Boettiger, and H. Holtzer (1983) Altered cell spreading in cytochalsin B: A possible role for intermediate filaments. Mol. Cell. Biol. 3: 113-125.

Mobley, W. C., A. Schenker, and E. M. Shooter (1976) Characterization and isolation of proteolytically modified nerve growth factor. Biochemistry 15: 5543-5551.

Moss, T. H., and S. J. Lewkowicz (1983) The axon reaction in motor and sensory neurones of mice studied by a monoclonal antibody marker of neurofilament. J. Neurol. Sci. 60: 267280.

Pruss, R. M., R. Mirsky, M. C. Raff, - Irpe, A. J. Dowding, and B. H. Anderton (1981) All cle f intermediate filaments share a common antigenic uecrminant defined by monoclonal antibodies. Cell 27: 419-428.

Quinlan, R. A., and W. W. Franke (1982) Heteropolymer filaments of vimentin and desmin in vascular smooth muscle tissue and cultured baby hamster kidney cells demonstrated by chemical crosslinking. Proc. Natl. Acad. Sci. U. S. A. 79: $3452-3456$.

Schlaepfer W. W. (1983) Neurofilaments of mammalian peripheral nerve. In Neurofilaments, C. A Marotta, ed., pp. 5785, University of Minnesota Press, Minneapolis.

Sharp, G., M. Osborn, and K. Weber (1982) Occurence of two different intermediate filament proteins in the same filament in situ within a human glioma cell line: An immunoelectron microscopical study. Exp. Cell Res. 141: 385-395.

Stallcup, W. B., L. S. Arner, and J. M. Levine (1983) An antiserum against the $\mathrm{PC} 12$ cell line defines cell surface antigens specific for neurons and Schwann cells. J. Neurosci. 3: $53-68$.

Tapscott, S. J., G. S. Bennett, and H. Holtzer (1981) Neuronal precursor cells in chick neural tube express neurofilament proteins. Nature 292: 836-838.

Trojanowski, J. Q., M. A. Obrocka, and V. M. - Y. Lee (1983) A comparison of eight different chromogen protocols for the demonstration of immunoreactive neurofilaments or glial filaments in rat cerebellum using the peroxidase-antiperoxidase method and monoclonal antibodies. J. Histochem. Cytochem. 31: 1217-1223.

Virtanen, I., V. -P. Lehto, E. Lehtonen, T. Vartio, S. Stenmon, P. Kurki, O. Wager, J. V. Small, D. Dahl, and R. A. Badley (1981) Expression of intermediate filaments in cultured cells. J. Cell Sci. 50: 45-63.

Wais-Steidler, C., P. A. M. Eagles, D. S. Gilbert, and J. M. Hopkins (1983) Structural similarities and differences amongst neurofilaments. J. Mol. Biol. 165: 393-400. 